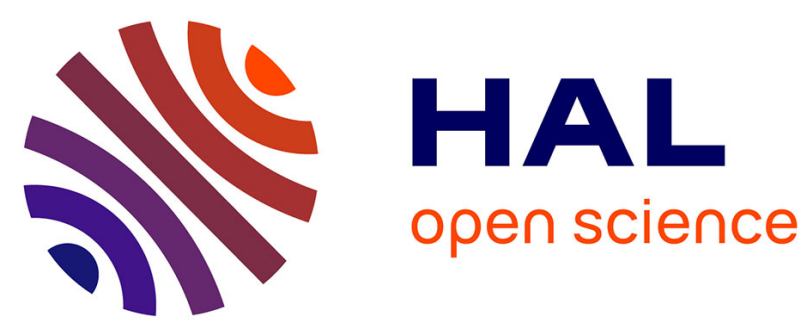

\title{
Gravity wave spectra in the middle atmosphere as observed by Rayleigh lidar
}

Richard Wilson, Alain Hauchecorne, Marie-Lise Chanin

\section{To cite this version:}

Richard Wilson, Alain Hauchecorne, Marie-Lise Chanin. Gravity wave spectra in the middle atmosphere as observed by Rayleigh lidar. Geophysical Research Letters, 1990, 17 (10), pp.1585-1588. 10.1029/GL017i010p01585 . insu-03130581

\section{HAL Id: insu-03130581 https://hal-insu.archives-ouvertes.fr/insu-03130581}

Submitted on 4 Feb 2021

HAL is a multi-disciplinary open access archive for the deposit and dissemination of scientific research documents, whether they are published or not. The documents may come from teaching and research institutions in France or abroad, or from public or private research centers.
L'archive ouverte pluridisciplinaire HAL, est destinée au dépôt et à la diffusion de documents scientifiques de niveau recherche, publiés ou non, émanant des établissements d'enseignement et de recherche français ou étrangers, des laboratoires publics ou privés. 


\title{
GRAVITY WAVE SPECTRA IN THE MIDDLE ATMOSPHERE AS OBSERVED BY RAYLEIGH LIDAR
}

\author{
R. Wilson, A. Hauchecorne, M.L. Chanin \\ Service d'Aéronomie du CNRS, Verrieres le Buisson Cedex, France
}

\begin{abstract}
Rayleigh lidar offers the unique ability to make routine measurements of temperature (or density) mesoscale fluctuations in the 30 to $75 \mathrm{~km}$ altitude range. Power spectra of density and temperature fluctuations, versus vertical wavenumber and (apparent) frequency, are derived from lidar measurements. These spectra are interpreted in the framework of the gravity wave theory. Power spectral density increases from the stratosphere to the mesosphere in the entire accessible spectral range. Comparison with vertical spectra obtained with various sounding systems indicates a dispersion of the temperature power spectra in the stratosphere as an increase with altitude of the ratio of potential to kinetic energy.
\end{abstract}

\section{Introduction}

Experimental evidence indicates a large similarity, in both shape and amplitude, in the power spectral density (PSD) of the wind and temperature mesoscale fluctuations in the middle atmosphere. Wind and temperature (or density) fluctuations data have been collected in the middle atmosphere with various sounding systems: mainly by balloon borne instruments, radar, lidar and rocket sounding. Nevertheless, the altitude range from 30 to $70 \mathrm{~km}$ is still unexplored, the wind and temperature fluctuations being obtained mainly by sporadic rocket soundings. Rayleigh lidar offers the unique feature of routine measurement in this altitude domain. There are now two operating Rayleigh lidars in southem France: at the Observatoire de Haute Provence (OHP) in the foothills of the Alps $\left(44^{\circ} \mathrm{N}, 6^{\circ} \mathrm{E}\right)$, and at Biscarosse (BIS) on the Atlantic coast $\left(44^{\circ} \mathrm{N}, 1^{\circ} \mathrm{W}\right)$. Data from these two sites are used in this study.

The nature of the mesoscale fluctuation field in the middle atmosphere is controversial. Van Zandt [1982] showed that the observed atmospheric spectrum of the mesoscale fluctuations can result, as in the ocean, from an internal gravity wave field, the so-called "universal spectrum". Another interpretation of the mesoscale variability of the atmosphere has been advanced by Gage [1979] who suggests that the horizontal wavenumber and frequency spectra of the horizontal wind fluctuations could result from two-dimensional (2D) turbulent eddies. No conclusion on the nature of the mesoscale fluctuations can be made from the measure of a single atmospheric parameter because both theories lead to similar predictions for some of the spectral characteristics of the motions. However, even if these two mechanisms coexist in the amosphere, the density or temperature fluctuations observed by Rayleigh lidar are associated with vertical displacement of the atmospheric fluid and thus should be less contaminated by $2 \mathrm{D}$ turbulence than horizontal wind measurements (pure 2D turbulence is constrained to horizontal planes). Therefore, the smallest vertical scale observable by Rayleigh lidar being of roughly one $\mathrm{km}$, an a priori hypothesis of no contamination by $2 \mathrm{D}$ turbulence is made. The lidar data are thus interpreted in the framework of the gravity wave theory.

The spectral characteristics of the mesoscale density (or temperature) fluctuations in the stratosphere and mesosphere,

Copyright 1990 by the American Geophysical Union.

Paper number 90GL01525

$0094-8276 / 90 / 90 G L-01525 \$ 03.00$ versus vertical wavenumber and apparent frequency, from a data set obtained by Rayleigh lidars, are presented. These spectra are then compared with temperature and horizontal wind spectra obtained by various sounding systems.

\section{Data analysis}

The backscattered light from a pulsed laser beam sent vertically into the atmosphere is due, in the absence of aerosols, to Rayleigh scattering from neutral molecules and is thus directly proportional to the atmospheric density, $p$. The relative density fluctuations, $p / p_{0}$, are estimated as the first order perturbation term of the vertical profile of $\log (\rho)$. The absolute temperature can be deduced from the relative density measurements by using the ideal gas law, assuming hydrostatic equilibrium, and by fitting the temperature profile at the top of the measurement, i.e. where the statistical error on the number of counted photons is $15 \%$, typically above $80 \mathrm{~km}$ (for more details see Chanin and Hauchecome [1984]).

From the mean density, $\rho_{0}$, estimated by low pass filtering, the static stability (characterized by the Brunt-Väisälä frequency $N$ ) of the atmosphere is deduced. The available potential energy per unit mass, $E_{p}$, associated with a vertical displacement $\xi$ of an air parcel is given by:

$$
E_{p}=\frac{1}{2} N^{2} \xi^{2}=\frac{1}{2}\left(\frac{g}{N}\right)^{2} \overline{\left(\frac{p^{2}}{p_{0}}\right)^{2}}
$$

High resolution measurements are performed to study gravity waves: the vertical integration is $300 \mathrm{~m}$ and the temporal integration $15 \mathrm{~min}$. The altitude domain is divided as follows: the upper stratosphere, from 30 to $45 \mathrm{~km}$, the lower mesosphere, from 45 to $60 \mathrm{~km}$, and the middle mesosphere, either 55 to $70 \mathrm{~km}$ or 60 to $75 \mathrm{~km}$.

The perturbation temperatures or densities, from which vertical wavenumber spectra are derived, are extracted by subtracting from the raw vertical profile (or from its logarithm) a third order polynomial fit applied to the $15 \mathrm{~km}$ altitude domain. In the case of frequency analysis a linear trend is removed from the raw data at a given altitude level. In order to reduce boundary effects, a Hanning window is applied to the spatial or temporal series, the spectrum being then normalized to the variance of the raw data. The mean vertical spectrum is averaged over all the spectra resulting from 15 min integration time of the signal obtained during a given period, whereas the mean frequency spectrum is averaged over the altitude levels in a given height range. The $2 \mathrm{D}$ spectra are obtained by applying a frequency Fourier Transform over the successive vertical wavenumber spectra. The 2D spectrum thus describes, for each wavenumber, the phase evolution of the sinusoidal functions, Fourier Transforms of the vertical profiles (resulting from 15 min integration time) of the density perturbations. The zero frequency term of the $2 \mathrm{D}$ spectrum represents the PSD of the fluctuations of periods larger than the measurement duration, such fluctuations being vertically resolved. The integration over frequencies of the 2D spectrum gives the 1D spectrum (versus vertical wavenumbers).

For the vertical wavenumber spectra, the perturbations are defined as $\left(g / N^{2}\right)\left(\rho^{\prime} / \rho_{0}\right)$ in order to get a comparison with respect to the saturation spectral limit independent of the mean 
static stability and temperature conditions (see eq. 2). The perturbations, in the frequency decomposition, are defined as $(\mathrm{g} / \mathrm{N})\left(\rho^{\prime} / \rho_{o}\right)$, whose variance is simply twice the available potential energy. It has been verified that spectra obtained from density or temperature measurements give identical results.

\section{Power spectra of the normalized density fluctuations in the stratosphere and mesosphere}

The PSD of the density fluctuations is interpreted in the framework of the gravity wave theory. In order to discuss the following results, we first briefly review the theoretical predictions about the power spectra of saturated density (or temperature) fluctuations. Furthermore, the comparison of density and horizontal wind spectra must necessarily rely on a spectral modelling of the wave field.

Using dimensional analysis, Dewan and Good [1986] and Smith et al. [1987] argued that the convective saturation of the wave motions lead to a relative density (or temperature) PSD versus vertical wavenumber, $m$, of the form:

$$
F_{\rho^{\prime} / \rho_{0}}(m) \cong F^{S_{T} / T_{0}}(m)=\alpha \frac{N^{4}}{g^{2} m^{3}}
$$

The index $s$ indicates saturation and $\alpha$ is a coefficient of the order of unity. Smith et al. [1987] estimate the spectral limit due to convective saturation process assuming a vertical wavenumber spectrum of the form:

$$
\mathrm{F}_{\rho^{\prime} / \rho \mathrm{o}}(\mathrm{m}) \sim \frac{1}{1+(\mathrm{m} / \mathrm{m} *)^{3}}
$$

where $m *$ is a characteristic wave number. Integrating over wavenumbers, we obtain:

$$
F_{\rho^{\prime} / \rho 0}(m)=\frac{N^{2}}{10 m^{3}}
$$

This estimate depends upon the chosen shape of the vertical spectrum (eq. 3) and upon the spectral width of the saturated wave field, expressed by $\ln \left(m * / m_{c}\right)$, where $m_{c}$ is the largest wavenumber contributing to saturation. The ratio $m * / m_{c}$, assumed to be invariant with altitude, is equal to 200 . The frequency and vertical wavenumber spectra are independent. Furthermore, the PSD in the saturated range is independent of the frequency energy distribution due to the hypothesis that convective instabilities are the only saturation mechanism.

The normalized density fluctuations PSD versus vertical wavenumber in the upper stratosphere, lower mesosphere and middle mesosphere, are shown in figure 1 . A straight line $1 / 2 \mathrm{~m}^{3}$ is plotted for a direct comparison with the saturation limit (eq.2). These spectra are monthly mean, averaged over 503 individual spectra (resulting from $15 \mathrm{~min}$ integration time of the lidar signal), obtained during January 1989 at Biscarosse. The shape of these spectra remains very similar in the three height ranges, especially for low wavenumbers, the slope being close to -2 for vertical scales larger than 3 or $4 \mathrm{~km}$.

In the upper stratosphere, from 30 to $45 \mathrm{~km}$, the slope of the high wavenumber portion of the spectrum, for wavelengths smaller than $3 \mathrm{~km}$, is close to -3 as predicted by the saturation theory, whereas the PSD is a factor of 2 below the convective limit of eq. $4(\alpha-1 / 20)$.

In the lower mesosphere (45 to $60 \mathrm{~km}$ ) the PSD is larger than in the stratosphere in the entire accessible spectral range. The high wavenumber portion of the spectrum is close to the saturation limit (eq.4) even if slightly larger $(1 / 5<\alpha<1 / 4)$.

In the middle mesosphere (55 to $70 \mathrm{~km}$ ) the PSD is again larger than at lower levels. The PSD is close to $\mathrm{N}^{2} / 2 \mathrm{~m}^{3}$ $(1 / 3<\alpha<1 / 2)$ for vertical wavelengths smaller than $4 \mathrm{~km}$.

PSD versus apparent frequency of the normalized density fluctuations are shown in figure 2. A -5/3 slope straight line is also plotted for comparison with proposed power law. These spectra are averaged over 5 long nights of measurements (11.5 hours), obtained during January 1989 at BIS. The spectral index $p$ (the mean slope of the frequency spectrum), close to -2 , does not change significantly from the stratosphere to the mesosphere. Nevertheless the contribution of large period modes (larger than 3 hours) seems to be comparatively more important in the stratosphere than at higher levels.

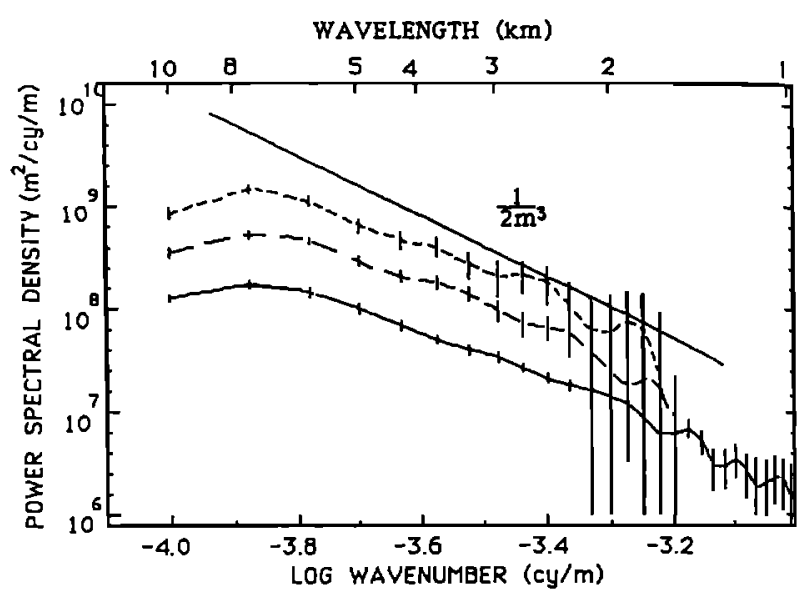

Fig. 1 : Power spectral density, versus vertical wavenumber, of the relative density fluctuations scaled by $g / \mathrm{N}^{2}$, in the stratosphere $(30-45 \mathrm{~km})$ [- $]$, lower mesosphere $(45-60$ $\mathrm{km})[--]$ and middle mesosphere $(55-70 \mathrm{~km})[--]$. A straight line $\mathrm{N}^{2} / 2 \mathrm{~m}^{3}$ is plotted for comparison.

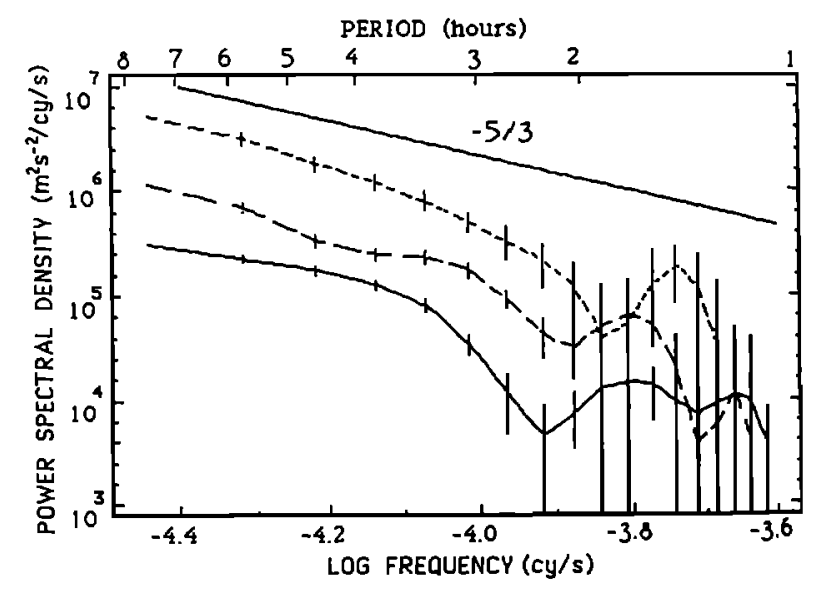

Fig. 2 : Power spectral density versus apparent frequency of the normalized density fluctuations observed in the stratosphere [-], lower [- -$]$ and middle mesosphere [- - ]. A straight line, of $-5 / 3$ slope, is plotted for comparison.

The 2D spectra, versus vertical wavenumber and apparent frequency (figure 3), averaged over the same nights as the frequency spectra, reveal a change with altitude of the wave's characteristics. In the upper stratosphere $(30-45 \mathrm{~km})$ most of the energy content (63\%) lies in stationary modes (or at least of larger period than the measurement time). In the mesosphere $(45$ to $60 \mathrm{~km}$ and 55 to $70 \mathrm{~km}$ ) the "stationary" modes are relatively much weaker than in the stratosphere (18 and $17 \%$ of the energy content respectively), indicating a strong damping of low frequency waves in the mesosphere. The existence of long period waves is confirmed by timeheight plots of the temperature fluctuations which show quasi stationary oscillations in the stratosphere. 
The observed increase with altitude of the normalized density PSD is clearly in disagreement with the spectral limit advanced by Smith et al. [1987] (eq.4). The power spectra are not scaled as $\mathrm{N}^{4}$ from the stratosphere to the mesosphere. The disagreement could be due to some simplifications in the spectral modelling of the wave field, mainly to the neglect of inertial effects. The estimate of the saturation spectral limit from convective instabilities induced by the wave field does not depend explicitly upon the frequency energy distribution. The ratio $\mathrm{m}_{\mathrm{c}} / \mathrm{m}$ * may also vary significantly from the stratosphere to the mesosphere where the outer scale of turbulence $m_{c}^{-1}$, could reach a few hundreds meters [Hocking, 1985] and $\mathrm{m}^{-1}$ is observed to be of the order of $10 \mathrm{~km}$. Furthermore, the assumption of a complete separability of the frequency and wavenumber spectra may be crude. Therefore, it is difficult to evaluate the effects of any change in the frequency distribution of the wave field (such as the damping of inertio-gravity waves in the mesosphere as suggested by the 2D spectra). This hypothesis call for further theoretical investigations.

$$
\begin{aligned}
& \text { BIE JAN } 89 \\
& 30-45 \mathrm{~km} \\
& \text { SE PE = } 063 \\
& \left(\mathrm{~m}^{3} \mathrm{~s}\right) \\
& \text { ABOVE } 130 \\
& 125-130 \\
& 1213-125 \\
& 116-120 \\
& 111-116 \\
& 106-111 \\
& 102-166 \\
& 97-102 \\
& 92-97 \\
& 88-92 \\
& \square \text { RELON } 88
\end{aligned}
$$

a
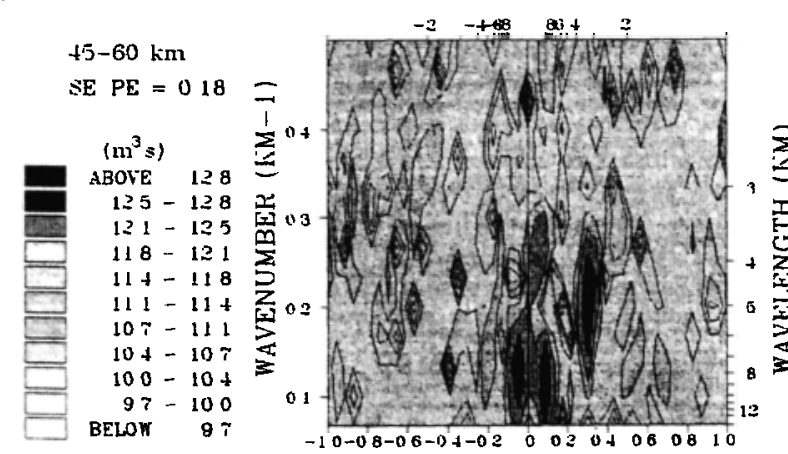

b
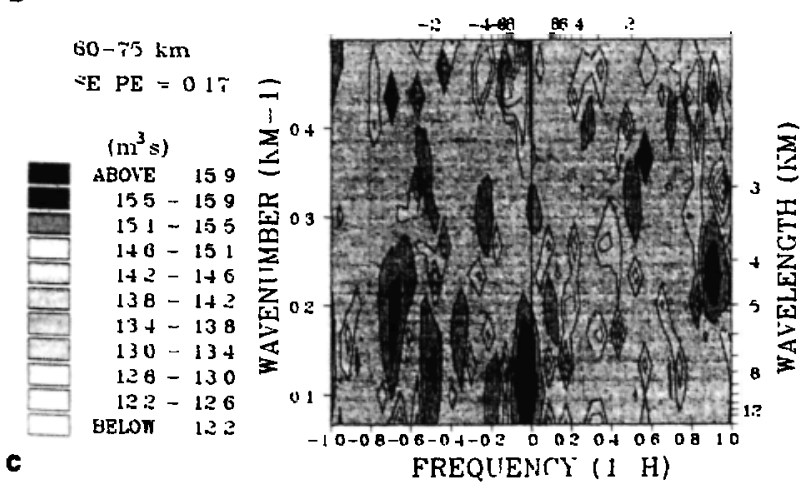

Fig. 3 : Two-dimension power spectra, function of the vertical wavenumber and apparent frequency, of the normalized density perturbations, in the stratosphere (a), lower (b) and middle mesosphere (c).

\section{Comparison with temperature and horizontal wind spectra}

Temperature data have been obtained in the lower stratosphere by mean of balloon borne instruments. Power spectra of relative temperature fluctuations $\left(T^{*} / T_{0}\right)$ resulting from balloon measurements during November [Fritts et al., 1988] and May [Sidi et al., 1988] in the 20.5-30 and 12-18 $\mathrm{km}$ height range respectively, and two lidar spectra (January and September 1989 at Biscarosse in the $30-45 \mathrm{~km}$ altitude domain) are superposed in figure 4 . The monthly means of January and September have been chosen because they are respectively representative of strong and weak wave activity, the seasonal variability of the wave activity being clearly annual in the stratosphere [Wilson et al., 1990]. Good agreement is found between these temperature spectra, taking into account the fact they have been obtained in various locations, time and altitude domains. Indeed, the spectral index is close to -3 in the high wavenumber portion of the spectra, the spectral amplitude ranging within a factor of four to five. Nevertheless, the variability from one spectrum to another appears to be significant. In the same height range the magnitude of the spectra obtained by lidar and in situ measurements ranges within a factor of 2 to 3 , the higher energy levels occurring in the lower stratosphere.

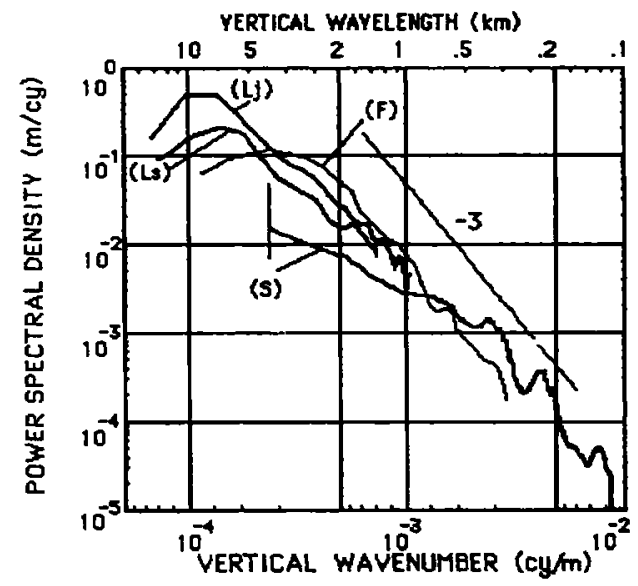

Fig. 4 : Power spectral density of the relative temperature perturbations obtained by Rayleigh lidar during the months of January (Lj) and September (Ls) 1989 at Biscarosse, and by balloon borne instruments by Fritts et al. [1988] (F) and Sidi et al. [1988], (S).

In figure 5, mean annual spectra of available potential energy in the upper stratosphere $(30-45 \mathrm{~km})$, lower mesosphere $(45$ $60 \mathrm{~km})$ and middle mesosphere $(60-75 \mathrm{~km})$ are plotted with kinetic energy spectra obtained from smoke trail data in the stratosphere [Dewan et al. 1984], by rocket soundings [Hass and Mayer, 1987] and MST radars [Smith et al., 1987] in the mesosphere, and by falling spheres in the lower thermosphere [Smith et al., 1987]. The kinetic energy spectra are deduced from the zonal or the meridional wind perturbations (or a mean of the two components), whereas the available potential energy spectra are defined as half of the PSD of the normalized temperature fluctuations $(g / N)\left(T / T_{0}\right)$. The potential energy spectra are averaged over the individual spectra obtained by Rayleigh lidar during four years at OHP, from 1984 to 1987. The PSD of available potential energy increases for all wavenumbers by roughly one order of magnitude from the stratosphere to the middle mesosphere, whereas the kinetic energy PSD does not change significantly in the same height range (scaled by $\mathrm{N}^{2}$ ). Such a growth, from the upper stratosphere to the lower mesosphere, of the temperature PSD has also been observed by Shibata et al. [1988] (on the basis of 13 Rayleigh lidar profiles), whereas Tsuda et al. [1989] 
have shown, from MST radar measurements, that horizontal wind PSD are scaled by $\mathrm{N}^{2}$ in the saturated range.

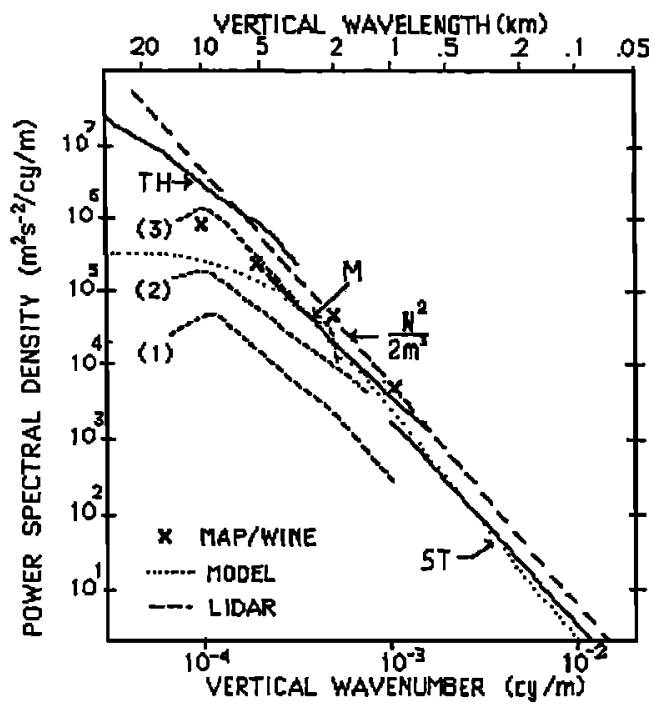

Fig. 5 : Power spectral density of potential and kinetic energy versus vertical wavenumber in various altitude ranges (adapted from Smith et al., 1987). TH and $M$ denote thermospheric and mesospheric horizontal velocity spectra [Smith et al., 1987]; ST denote stratospheric [Dewan et al., 1984]; X's represent a mean spectrum of the horizontal wind perturbations in the 30$64 \mathrm{~km}$ altitude range, resulting from rocket soundings during the MAP-WINE campaign [Hass and Mayer, 1987]; short dashed lines are potential energy spectra deduced from lidar measurements, (1) in the stratosphere, (2) in the lower mesosphere and (3) in the middle mesosphere.

There is thus a significant increase in the ratio of potential to kinetic energy from the stratosphere to the mesosphere. This ratio, in the framework of the gravity wave theory, depends upon the frequency distribution of the wave field. Such an increasing ratio suggests a change in the frequency distribution of the waves, i.e. relatively lower frequency waves in the stratosphere than in the mesosphere. Selective transmission due to critical lines, wave generation or damping processes could modify the energy distribution versus frequency of the wave field. This assumption is supported by the $2 \mathrm{D}$ spectra (figure 3) which indicate a dominant contribution of low frequency modes in the stratosphere and of relatively higher frequency modes at higher levels. Dynamical instabilities should then be the main saturation process in the stratosphere whereas convective instabilities could be relatively more important in the mesosphere. Nevertheless the possibility that other processes, such as 2D turbulence, contribute to the mesoscale atmospheric variability cannot be excluded, even though it is not possible to reach a definitive conclusion from such a heterogeneous data set.

\section{Conclusions}

Some main features of the spectral energy distribution of the density (or temperature) fluctuations in the middle atmosphere have been described from Rayleigh lidar measurements. The PSD of the density fluctuations is observed to increase with altitude in the accessible spectral range. A large contribution in the energy content of low frequency waves is observed in the stratosphere, these long period waves being strongly damped in the lower mesosphere. In the stratosphere, the temperature fluctuations PSD versus vertical wavenumber vary within a factor of four, in the high wavenumber portion of the spectrom (assumed to be saturated), depending on season and altitude. An increase with altitude of the ratio of potential to kinetic energy is observed. Changes in the frequency distribution of the wave field with location, time and altitude could be the reason for such a dispersion of the temperature PSD in the stratosphere and for the observed increase with altitude of the ratio of potential to kinetic energy.

\section{References}

Chanin M.L. et A. Hauchecorne, Lidar studies of temperatare and density using Rayleigh scattering, MAP Handbook. 13, 87-99, 1984

Dewan E.M., N. Grossbard, A.F. Quesada and R.E. Good, Spectral analysis of $10 \mathrm{~m}$ resolution scalar velocity profiles in the stratosphere, Geophys. Res. Lett. 11, 80-83, 1984. Correction, 11, 624, 1984.

Dewan E.M.and R.E. Good, Saturation and the "universal" spectrum for vertical profile of horizontal scalar winds in the stratosphere, J. Geophys. Res. 91, 2742-2748, 1986

Fritts D.C., T. Tsuda, T. Sato, S. Fukao and S. Kato; Observational Evidence of a Saturated Gravity wave Spectrum in the troposphere and lower stratosphere, $L$ Atmos. Sci. 45, 1741-1759, 1988

Gage K.S., Evidence for k-5/3 law inertial range in mesoscale two-dimensional turbulence, J. Atmos. Sci, 36, 19501954,1979

Hass $H$. and Meyer W. Gravity wave field above Andoya, L Atmos. Terr. Phys. 49, 705-712., 1987.

Hocking W.K., Measurements of turbulent energy dissipation rates in the middle atmosphere by radar technique: $A$ review, Radio Sci, 20, 1403-1422, 1985

Shibata T., S. Ichimori, T. Narikiyo and M. Maeda, Spectral analysis of vertical temperature profiles observed by a lidar in the upper stratosphere and the lower mesosphere, I. Meteorol. Soc. Japan 66, 1001-1005, 1988

Sidi C., J. Lefrère, F. Dalaudier and J. Barat, An improved atmospheric buoyancy wave spectrum model, L Geophys, Res., $93,774-790,1988$.

Smith S.A., D.C Fritts and T.E. Van Zandt: Evidence of a saturated spectrum of atmospheric gravity waves, $L$ Atmos. Sci 44, 1404-1010, 1987

Tsuda T., T. Inoue, D.C. Fritts, T.E. VanZandt, S. Kato, T. Sato and S. Fukao, MST radar observation of a saturated gravity wave spectrum, I_Amos.Sci, 46, 2440-2447, 1989

VanZandt T.E, A universal spectrum of buoyancy waves in the atmosphere, Geophys. Res. Lett. 2. 575-578, 1982

Wilson R., M.L. Chanin and A. Hauchecome: Gravity waves in the middle atmosphere observed by Rayleigh lidar. Part 2: climatology, submitted to J.Geophys. Res, 1990

R. Wilson, M.L. Chanin, A. Hauchecorne, Service d'Aéronomie du CNRS, BP3, 91371 Verrières-le-Buisson, France

(Received January 4, 1990 revised March 22, 1990; accepted May 16, 1990) 